\title{
Focus on challenges and advances in the treatment of patients with penetrating injuries
}

\author{
T. Lustenberger ${ }^{1} \cdot \mathbf{P}$. Talving ${ }^{2}$
}

Received: 6 November 2016 / Accepted: 10 November 2016 / Published online: 17 November 2016

(C) Springer-Verlag Berlin Heidelberg 2016

The incidence of penetrating injury varies widely across different continents and regions of the world. The management of patients with penetrating injuries is costly and poses multiple challenges, particularly so in low-volume settings, such as Europe, Australia, and Asia. Yet, it is crucial that penetrating injuries are managed somewhat differently from blunt mechanisms keeping focus on vascular and hollow viscus lesions. This special issue of the European Journal of Trauma and Emergency Surgery is dedicated to updates on epidemiology and management of penetrating trauma from low-volume penetrating trauma settings of Europe and Australia.

In the first paper, Störmann et al. [1] provide an epidemiological overview on penetrating injuries in a European urban area. In Western Europe, penetrating injuries are by far less frequent compared to countries, such as the US and South Africa. Nevertheless, in this study, approximately 9\% of all trauma room admissions were due to penetrating injury mechanisms, mainly caused by interpersonal violence or attempted suicide. The authors discuss the distribution of penetrating injuries and compare the specific injury patterns to blunt trauma mechanisms. Surgical procedures and outcomes are likewise addressed.

T. Lustenberger

tom.lustenberg@gmail.com

P. Talving

peep.talving@ut.ee

1 Department of Trauma, Hand and Reconstructive Surgery, University Hospital Frankfurt, Theodor-Stern-Kai 7, 60596 Frankfurt, Germany

2 North Estonia Medical Center, Tartu University Hospital, University of Tartu, Tartu, Estonia
The second article by Kevric et al. [2] from Australia focuses on the management of hemodynamically stable patients presenting with abdominal stab wounds. While there is a consensus on the management of hemodynamically unstable patients following penetrating abdominal injuries, the management of hemodynamically stable patients with abdominal stab wounds is a matter of lively debate. In recent years, selective non-operative management (SNOM) has gained popularity following numerous reports on its efficacy and safety published in the literature. Kevric and colleagues present their experience and management algorithm for stable patients with penetrating abdominal injuries. The authors clearly highlight, that compliance with an algorithm towards SNOM in these injuries is associated with lower rates of non-therapeutic laparotomies.

The systematic literature review by Narvestad et al. [3] summarizes eight European studies on emergency resuscitative thoracotomy (ERT) in patients with blunt and penetrating injuries. While the majority of manuscripts on ERT originate from the US and South Africa, little is known about the outcome of ERT in the European settings. The present article reviews collective experience and outcomes of ERT in European civilian trauma patients. The authors report a $25 \%$ favorable outcome following emergency department thoracotomy. Not surprisingly and as known from previous studies, patients with penetrating trauma requiring ERT have a significantly better outcome compared to those following blunt mechanisms. Nevertheless, in this report, the accumulated survival rates for blunt trauma were considerably higher than previously reported, inquiring the poor perspectives of ERT after blunt injury.

Finally, the article by Branco and DuBose [4] reviews the current literature on endovascular management of penetrating arterial injuries, focusing on resuscitative 
endovascular balloon occlusion of the aorta (REBOA) and axillo-subclavian injuries. Endovascular procedures have been increasingly utilized in the management of penetrating injuries over the last decade. For patients in hemorrhagic shock and imminent cardiovascular collapse, REBOA has emerged as an alternative to open aortic occlusion. By inflating a balloon in the appropriate segment of the aorta, thus providing an endovascular proximal control to massive bleeding from non-compressible areas of the body, such as the chest, abdomen, or pelvis, REBOA is an attractive temporary intervention prior to definitive surgical management. Branco et al. analyze seven previous studies on REBOA following penetrating trauma and conclude that it is a safe and effective alternative to open thoracotomy in these instances. In the second part of their review, the authors discuss the management of penetrating axillo-subclavian injuries comparing open and endovascular repair. These injuries, likewise, carry high morbidity and mortality rates due to massive hemorrhage and complex open exposures.

This special issue of the EJTES gives you insight in current concerns and advances made in the treatment of patients with penetrating trauma. The presented articles reflect a snapshot of the current "state of the art" treatment as well as challenges, problems, and limitations in the management of these injuries. As guest editors of this issue, we hope you enjoy reading our selection of topics around penetrating injuries; further research is certainly needed to continuously improve patient's safety and outcome.

\section{Compliance with ethical standards}

The authors comply with the ethical guidelines for authorship and publishing in the European Journal of Trauma and Emergency Surgery. This article does not contain any studies with human or animal subjects performed by the authors.

Conflict of interest Thomas Lustenberger and Peep Talving declare no conflict of interest in relation to this work.

\section{References}

1. Störmann P, Gartner K, Wyen H, et al. Epidemiology and outcome of penetrating injuries in a Western European urban region. Eur J Trauma Emerg Surg. 2016. doi:10.1007/ s00068-016-0630-4.

2. Kevric J, O'Reilly GM, Gocentas RA, et al. Management of haemodynamically stable patients with penetrating abdominal stab injuries: review of practice at an Australian major trauma centre. Eur J Trauma Emerg Surg. 2016. doi:10.1007/ s00068-015-0605-x.

3. Narvestad JK, Meskinfamfard M, Søreide K. Emergency resuscitative thoracotomy performed in European civilian trauma patients with blunt or penetrating injuries: a systematic review. Eur J Trauma Emerg Surg. 2016. doi:10.1007/ s00068-015-0559-z.

4. Branco BC, DuBose JJ. Endovascular solutions for the management of penetrating trauma: an update on reboa and axillo-subclavian injuries. Eur J Trauma Emerg Surg. 2016. doi:10.1007/ s00068-016-0739-5. 